\title{
Routing Mechanisms for Multi-hop Cellular Communications in the WINNER Air Interface
}

\author{
Keivan Navaie ${ }^{1}$, Yajian Liu $^{2}$, Mohammad Abaii ${ }^{2}$, Adrian Florea ${ }^{1}$, Halim Yanikomeroglu ${ }^{1}$, Rahim Tafazolli ${ }^{2}$ \\ ${ }^{1}$ Broadband Communications and Wireless Systems \\ (BCWS) Centre, Department of Systems and Computer \\ Engineering, Carleton University, Ottawa, Canada \\ E-mail: \{keivan,aflorea,halim\}@sce.carleton.ca \\ ${ }^{2}$ Centre for Communication Systems Research,
School of Electronics and Physical Science,
University of Surry, Guildford, Surry, UK
E-mail:\{Y.Liu,M.Abaii,R.Tafazolli\}@surrey.ac.uk
}

\begin{abstract}
In this paper we present network-centric and usercentric routing mechanisms designed for the Wireless world INitiative NEw Radio (WINNER) multi-hop cellular air interface. We study the pros-and-cons of each mechanism and describe the interactions between routing and main Radio Resource Management (RRM) functionalities.
\end{abstract}

Index Terms-Beyond 3G, cellular coverage, network capacity, multi-hop cellular networks, fixed-relay, routing, radio resource management.

\section{INTRODUCTION}

There has been an increasing interest in the infrastructurebased wireless multi-hop networks in academia, industry, and standardization bodies and it seems that relay-based deployment concepts will play an important role in the costeffective provision of very high data rates in an almostubiquitous manner. Cost-effectiveness is a crucial point for the success of Beyond $3^{\text {rd }}$ Generation (B3G) cellular networks.

For the next generation of cellular networks, relay-based multi-hop cellular deployment has been considered as a potential air interface technology by Wireless World Research Forum (WWRF) as well as the Wireless world INitiative NEw Radio (WINNER) [1].

The WINNER project was initiated to conduct research toward developing a ubiquitous radio access technology for B3G wireless communications. This project is an EU-funded consortium of more than 40 partners from both academia and industry.

The overall goal of the WINNER project is to develop a new radio access technology whose parameters can be adapted to a comprehensive range of mobile communication scenarios from short-range to wide-area based on the $\mathrm{B} 3 \mathrm{G}$ system requirement described in ITU-R standard M.1645 [2]. Such system should support potential new frequencies above $2.7 \mathrm{GHz}$ as well as very high data bit-rates. Using conventional cellular architecture for B3G systems requires very small cells thus large number of base-stations (BSs). Therefore, novel deployment concepts are required.

It has been shown that relaying techniques are able to provide cost-effective and flexible radio network deployment
[3]-[6]. In the WINNER radio access, Fixed Relays (FRs) installed in the network coverage area have been considered as an integral part the air interface technology [5]. In such a deployment, routing in both uplink and downlink is a crucial Radio Resource Management (RRM) functionality.

The objective of the routing in the WINNER air interface is to optimize the system performance. Routing also provides inputs to (or has interactions with) other RRM functions. In this paper, we present the candidate routing mechanisms for the WINNER air interface. We also discuss the interactions between the routing and three main WINNER RRM functionalities.

In Section II we introduce the candidate routing architectures for the WINNER air interface including networkcentric and user-centric routing. We also investigate the prosand-cons of these two routing architectures. In Section III the interactions between the routing mechanism and other RRM functionalities are studied. Finally, we conclude the paper in Section IV.

\section{WINNER ROUTING ARCHITECTURES}

The WINNER air interface technology is based on multihop cellular deployment in which fixed-relays located in the coverage area of the cellular network are used to extend either network coverage or capacity distribution [5]. Routing is performed per user; however per-flow routing can be also considered as an option to facilitate advanced network-wide RRM schemes. Communication between BS and user terminals (UTs) follows a star topology, i.e., each FR communicates with only one BS. Packets can follow different routes in uplink and downlink; therefore, separate routing should be implemented for the uplink and the downlink. Finally, packet forwarding for an assigned route is performed in the Medium Access Control (MAC) layer.

From the architectural perspective, two basic routing strategies are envisioned including network-centric and usercentric. In the following section we describe these two routing strategies.

\section{A. Network-centric Routing Architecture}

In the network-centric routing architecture (Fig. 1) the 


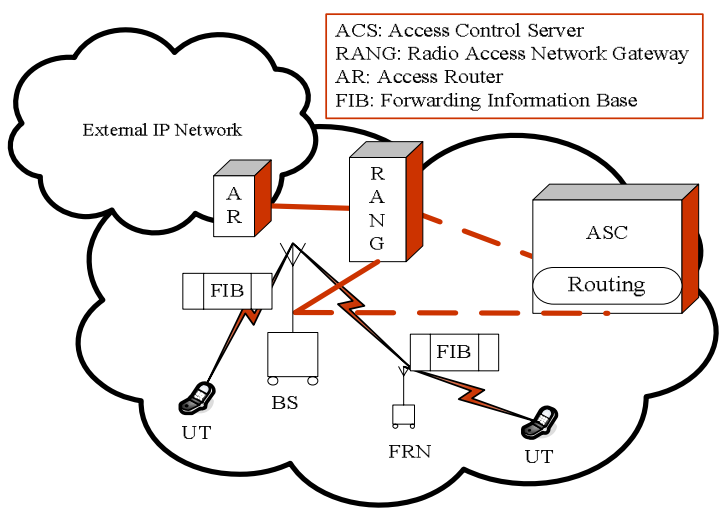

Figure 1: Network-centric routing architecture in the WINNER air interface.

routing functionality implemented at the Access Control Server (ACS), which is a network logical node responsible for controlling the access to the air interfaces radio resources. Each ACS manages a number of BSs and FRs. The routing information is then distributed in a data base, Forwarding Information Base (FIB) which is located in the corresponding BSs and FRs. The information in this database is then utilized for packet forwarding in the MAC layer.

Network-centric routing consist of four basic functional modules including Route Discovery, Proactive Routing, In-use Routes Updating, and Candidate Routes Database (see Fig. 2).

The Route Discovery module obtains a set of initial candidate routes upon receiving Initial Cell/Mode Selection request during the flow establishment initiated by either UT or BS. Candidate Routes Database holds up-to-date list of candidate routes for all active users.

In order to keep track of the quality of the candidate routes in the database Proactive Routing module is executed either periodically or on-demand.

For each entry in the candidate route database an in-use flag is considered which indicates whether that route is used by the corresponding flow or not. The main task of the In-use Routes Updating module is to update the "in-use" flag of corresponding routes in the database under the requests of other RRM functions. Each active flow has one in-use route.

The required information for Route Discovery and Proactive Routing modules are provided by MAC Control Feedback and Load Control signals. The operating time-scale of the Proactive Routing module is fairly slow e.g., once per several seconds. The time-scale of operation may be adjusted based on the users' mobility pattern and/or their spatial traffic characteristics. The actual route is then selected and/or assigned from the set of candidate routes by other RRM functions such as Admission Control and Hand-over.

After selecting a route a request is sent to In-use Route Updating module to update the in-use flag of corresponding routes in Candidate Route Data-base. The list of in-use routes is then sent by Forwarding Information Distribution signal to the corresponding BSs and FRs to update their corresponding

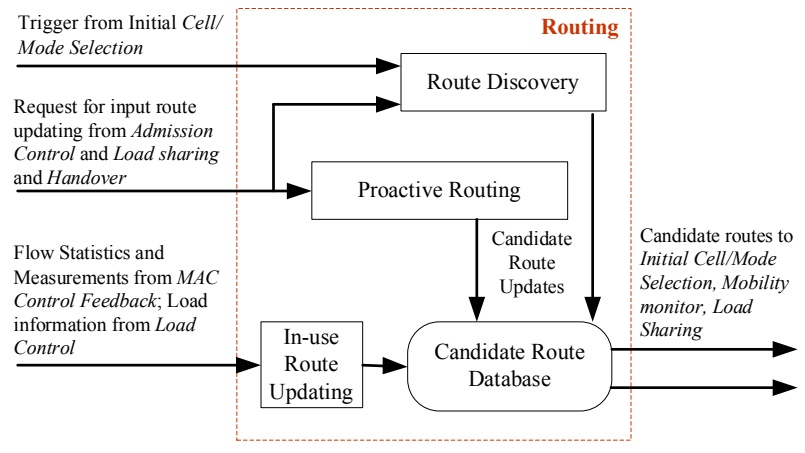

Figure 2: The internal structure of the routing functional module

FIBs. The typical components within an entry in FIB database are e.g., the destination address (or flow ID) and the address of the next participating hop in the route.

Network-centric routing requires channel status information between users and BSs/FRs as well as the loading status of BSs and FRs. In the WINNER air interface these measurements are already available in ACS to be used by other RRM functions such as hand-over, admission control and load-sharing.

A major advantage of network-centric routing is that the cross-optimization can be performed in order to improve the overall system performance. As a result, the network-centric route calculation algorithm usually involves a degree of algorithmic complexity. Nevertheless, because of the powerful process capability of the central node, sophisticated routing algorithms are feasible to be implemented. Moreover, heuristic algorithms can also be devised to reduce the computational complexity of course with the compromise on performance degradation.

The route calculation algorithm also needs some measurements as input including e.g., channel qualities between users and BSs/FRs, and the loading status of BSs and FRs. Fortunately, they are usually available in the central node to be used by other RRM functions such as handover, admission control and load sharing. To conclude, the centralized routing strategy is not likely to induce much extra complexity due to the signaling overhead of input information gathering.

\section{B. User-Centric Routing Architecture}

In the user-centric routing architecture, the routing decision is made by UT based on the link cost information signal broadcasted by the FRs and BSs over their broadcast channels (BCHs).

Link cost information for the BS-FR link indicates the radio channel status (e.g., path-loss, interference) as well as the BS or FR utilization (e.g., their traffic load). For the BS-UT link UT can extract the radio channel status from received signal measurements; therefore in this case the link cost only indicates the utilizations of the BS.

The UTs extract the information in the $\mathrm{BCH}$ and evaluate the best access point. Implicitly, the selection of the best 
server also results in a route to be followed toward the access point into the core network. Note that in user-centric routing the selection of the best server is made based on both radio channel status and traffic load.

Based on the downlink pilot signal measurements, the UT evaluates the cost of the air interface link UT-FR for received pilots. From BCH the UT also extracts the costs related to the FRs utilization as well as the cost of the FR-BS link. By selecting the FR with the lowest total cost the UT also selects an appropriate route. Hence, it can be seen that the UT selects not only the best server, but actually the best access route. The algorithm can be enhanced to consider additional parameters such as the user subscription attributes.

The radio resources in each cell including the $\mathrm{BS}$ and the first tier of FRs are administered by the BS. Therefore, if the traffic load increases within the coverage area of a FR, the BS can assign it additional resources at the expense of other FRs in the cluster. The BS do not have visibility into resource assignment/scheduling within a FR. Similarly, the assignment of resources for competing BSs will be arbitrated by the central ACS entity. The ACS will not have visibility into cluster level resource assignments, which are controlled by the BSs.

In the above user-centric routing architecture, the signaling overhead related to the routing is limited to the cost information broadcasted by BCHs. UTs then make routing decision based on a simple decision making procedure to find the route with the lowest cost. Therefore, the routing complexity is mainly a function of the number of received BCHs. For cases with more than two hops, signaling overhead is an important factor. In such cases the decision making procedure is also more complex.

\section{Routing implementation in the WINNER air interface}

The network-centric routing architecture is potentially more capable for network-wide performance optimization through sophisticated RRM schemes. However, the computational complexity and signaling overhead of network-centric routing should be carefully taken into consideration.

User-centric routing approach, on the other hand, reduces signalling overhead. Moreover, since the routing decision in this approach is made locally, user-centric approach react in responding to changing conditions in the air interface with a lower latency. The user-centric routing however requires a higher level of computational complexity in UTs. Such approach, because of its distributed nature, may also make implementation of the advanced traffic management functionalities harder than that of in the network-centric counterpart. The signalling overhead for user-centric approach is increased for cases with more than two hops.

In the following section we describe the interactions of the routing with the main RRM functionalities in the WINNER air interference, more details on these functionalities are presented in [5].

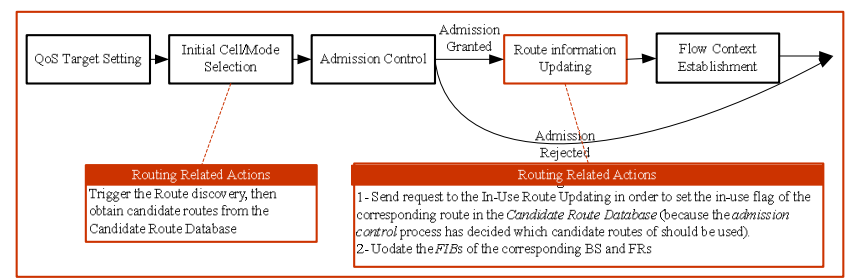

Figure 3: Interaction between Routing and Admission Control

\section{ROUTING INTERACTION WITH OTHER RRM FUNCTIONS}

\section{A. Interaction between routing and admission control}

In order to make an appropriate admission control decision for a service demanded by a user, the list of possible routes for that user is necessary. Therefore, prior to the Admission Control decision-making, Route Discovery module needs to be executed to update the candidate route database for that particular flow. If the flow is admitted, the in-use route for the flow is then chosen and Route Information Updating module is executed. In-use Route Updating module is also executed to set the in-use flag of the corresponding route in the Candidate Routes Database, and update the FIBs of the corresponding BSs and FRs (Fig. 3).

\section{B. Interaction between routing and hand-over}

Hand-over is often required for mobility management. In the WINNER, Mobility Monitor module keeps monitoring the quality of the candidate routes for individual users. Once the Mobility Monitor module finds the in-use route of a flow needs to be updated, it triggers the hand-over process. If the hand-over is successful, requests will be sent to the In-use Route Updating module in order to modify the in-use flag of corresponding routes in the Candidate Route Database. The FIBS of the corresponding BSs and FRs will be updated accordingly (Fig. 4).

\section{Interaction between routing and load-sharing}

Load-sharing in the WINNER is responsible for improving operational system performance by means of appropriately sharing among FRs and BSs. The Load-sharing module periodically checks the candidate routes of flows. If it is found beneficial for the system to change the route of a flow (e.g., cases in which re-routing can avoid overloading of some FRs or BSs), it will trigger the hand-over process to an adjacent FR or BS. The routing related actions after the hand-over are similar to those explained in the Section III.B (Fig. 5).

\section{CONCLUSIONS}

We propose network-centric and user-centric routing strategies for the WINNER air interface. Under networkcentric, route computation is performed in a central controller, which usually possesses powerful processing capabilities and at the same time has knowledge on the global network status. 


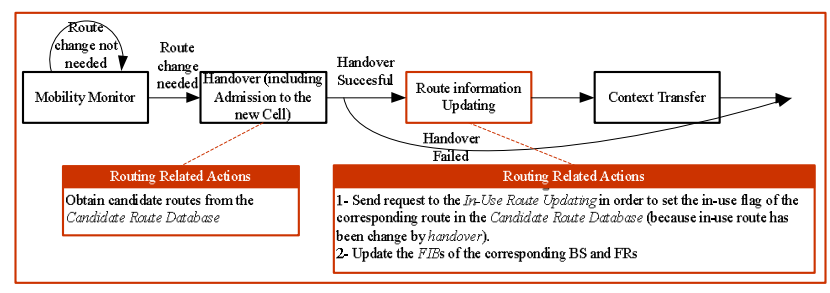

Figure 4: Interaction between Routing and Hand-over

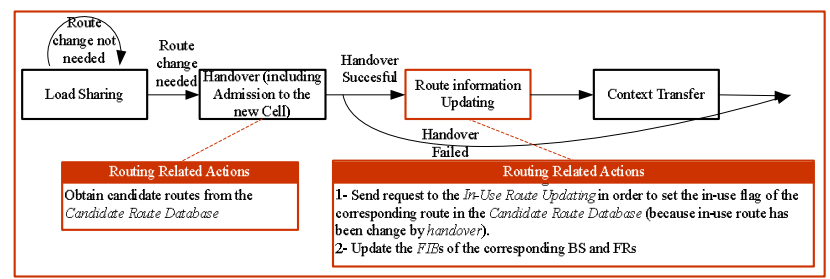

Figure 5: Interaction between Routing and Load-sharing

In user-centric routing, all network nodes between the source and the destination are involved in the route determination. This strategy can function when no central controller is reachable, but its performance is normally limited by the processing capabilities of the network nodes and its knowledge of the network status. In the WINNER air interface, both the network-centric and user-centric routing strategies are feasible to implement.

Since the objective of the routing function in the context of WINNER systems is to optimize system performance (e.g. throughput), instead of to maintain the network connectivity, the network-centric routing is potentially more capable of fulfilling this objective. However, the computational complexity and signaling overhead of network-centric approach should be taken into consideration. In the first phase of the WINEER project, maximum number of hops is envisioned to be two. Therefore, the signaling overhead and delay of the centralized strategy (caused by the information gathering) is acceptable. In addition, other RRM functions require lots of measurements, e.g., link qualities, some of these measurements can be shared with the centralized routing function, and thus further reduce its signaling overhead.

Use-centric routing approach reduces signalling overhead.
Since the routing decision in this approach is made locally, this approach can potentially react with a lower latency in responding to changing conditions in the air interface. The User-centric routing however may require a higher level of computational complexity in UTs. Such approach because of its distributed nature may also make implementation of the advanced traffic management functionalities harder than that of in the centralized architecture. The signalling overhead for this approach is significantly increased for cases with more than two hops and or meshing.

\section{ACKNOWLEDGMENT}

This work has been performed in the framework of the IST project IST-2003-507581 WINNER. The work of K. Navaie, A. Florea and H. Yanikomeroglu was supported by Special Research Opportunities (SRO) grant from Natural Sciences and Engineering Research Council (NSERC) of Canada. The work of Y. Liu, M. Abaii and R Tafazolli was partly funded by the European Union. The authors would like to acknowledge the contributions of their colleagues in WINNER WP3. The views expressed are those of the authors and do not necessarily represent the project.

\section{REFERENCES}

[1] www.ist-winner.org

[2] ITU Recommendation M.1645.

[3] "Description of identified new relay based radio network deployment concepts and first assessment", WINNER Deliverable D3.1, Nov. 2004. Available online: https://www.istwinner.org/publicdeliverables.htm

[4] K. Navaie, H. Yanikomerolgu, "Multi-route and Multi-user Diversity in Infrastructure-based Multi-hop Networks," in Cooperation in Wireless Networks: Principles and Applications, Editors: F. Fitzek and M. Katz, Springer, 2006.

[5] "Proposal of the best suited deployment concepts for the identified scenarios and related RAN protocols", WINNER Deliverable D3.5, Dec. 2005. Available online: https://www.ist-winner.org/publicdel iverables.htm

[6] Y. Liu, R. Hoshyar, X. Yang, R. Tafazolli, "On the Radio Resource Allocation in Enhanced Uplink UTRA-FDD with Fixed Relay Stations," in Proc. IEEE PIMRC'05, Sep. 2005. 